\title{
BUILDING BRIDGES OF UNDERSTANDING AND EMPOWERMENT BETWEEN SOCIETY AND RESEARCHERS
}

\author{
Michelle Rotchford Galloway, MPhil (fourn) \\ Media and Communications Manager, South African AIDS Vaccine Initiative (SAAVI)
}

Masikhulisane, meaning 'Let us grow together', is the new name of the revamped and refocused community involvement programme of the South African AIDS Vaccine Initiative (SAAVI). The programme aims to create an equal, meaningful partnership between community and scientists to facilitate AIDS vaccine research and promote overall understanding of medical research. As the programme manager Elise Levendal says: 'It's for us to bring AIDS vaccine researchers and communities closer to each other.'

Community preparatory work and educating communities about AIDS vaccines and clinical trials has been an important component of the initiative since it started in 1999. Since then, this work has received funding from the European Commission.

'It started off as a community preparation programme', says Elise. 'We have changed it into a community involvement programme because we think that people can be involved in many different ways.'

It's a challenging arena, and one that has required adaptation and refocusing over the years.

'I don't think this work is unique,', says Elise. 'In the 1980s in this country there was a big move towards community-based research. But what is particular to this programme is that it is a dedicated community programme for AIDS vaccine research and development.'

Among the questions that have arisen are which communities to focus on, how to work at a national level with limited resources, how to educate about vaccines when people don't have a basic understanding of HIVIAIDS, how to integrate your efforts with other educational initiatives, and, importantly, how to do all of this within an ethos that respects human rights.

Vaccine educator Petronella Goliath highlights the complexities. 'When you talk about community involvement, communities want to know what you mean - what level of community do you want to involve?'

'You also need to be really knowledgeable about HIV because sometimes when the group doesn't have the basic information you can spend the whole session on that and not even cover vaccines,' she continues. 'Then you have to go back and do a follow-up session on vaccines.'
'Most of our strategies are based around education, communication and training,' says Elise, 'but we also work within a human rights approach.'

Among the strategies employed are direct awareness raising and educational initiatives via workshops, information provision at local, national and international events, and the development of high-quality learning materials. Masikhulisane hopes to have its learning programme accredited and is working with the Standards Generating Body for Ancillary Health Care. The aim is to have standards generated for HIV/AIDS vaccine education and approved by the South African Qualifications Authority.

Elise explains that the programme works on a sectoral basis. 'The vaccine educators in each of the three provinces we are working in - Cape Town, Gauteng and KwaZulu-Natal, where there are already trial sites - have prioritised sectors. These could be traditional healers, the youth sector, community radio, for example.'

\section{ALL ABOUT TIMING}

'In terms of when to start with community education, what we have learnt is that there is never a right or wrong time. Some countries started with generic education and later wondered if they shouldn't have started with site specific. Other countries firmly believe that they should have started with generic education before going on to site specific. We are doing a bit of both. We started off with generic and moved into site specific - now we are doing both.'

'We believe that people retain the memory of the generic education,' she continues. 'It's not lost. When the trials start you do your site-specific education.'

The philosophy is not to influence people to be in favour of or against AIDS vaccine research but rather to share ideas and provide accurate information. 'We believe that when people have the education they will decide which role they want to have within vaccine research and development,' says Elise. 'There are different roles - it could be as a Community Advisory Group member, it could be as a trial participant, it could be educating other people about vaccines, it could be advocacy for vaccines. But communities and individuals must decide for themselves based on adequate information.' 
Interestingly, research done in Kenya has shown that the more information people have the less likely they are to participate in vaccine trials.

'That is worrying, of course,' says Elise, 'but it is only research from one country. We believe - but this is just an assumption - that the more education people get, the more informed people will become to take decisions about involvement in the vaccine development process.'

'It's all about getting accurate information and ideas out to people. And the education works both ways.

'It's a two-way process - we are not only taking scientific knowledge to communities but also taking community knowledge back to scientists', says Petronella.

'We want to ensure that both society and researchers learn together about human rights in medical research;' explains the programme's human rights advisor, Thomas Smit. 'Human rights in medical and related research would be concerned with both the development of a safe, affordable and effective vaccine, and with the human rights challenges posed by the development of such a vaccine. Human participation in clinical trials needs particular attention from a human rights perspective.'

'We want to build bridges of understanding and empowerment between society and researchers', he continues. 'When SAAVI initially engaged with human rights activists and ethicists there was a huge gap. That gap has narrowed - researchers no longer react as defensively as many did initially and society generally is starting to see more value in research. We need to ensure that those bridges are further developed and maintained as we undertake more and more relevant research especially in diseases that affect the developing world and our population.

'The fact that we come to people and educate them about this new process - it's a human right, education is a basic right', says Petronella.

'Although our work is conceptualised within AIDS vaccine work we see it as beneficial to all research, all clinical trials within the country,' says Elise.

A firm principle of the programme is that meaningful community involvement enhances and can even guide research processes, and there is definitely more of a culture of asking questions about research.

Some of the human rights issues that are important in vaccine development and will be further investigated include ensuring proper informed consent for participation; issues around the appropriateness of research populations, which also includes the involvement of children and adolescents in research ensuring broad-based gender and population issues around how to conduct trials appropriately in conditions of underdevelopment; and ensuring that society can engage in a meaningful way with medical research.

'Communities need to be learning about and addressing the human rights impact of medical research', says Thomas. 'We need to develop capacity together to ensure that research is developed and used for the benefit of all humanity and does not result in exploitation.'

'The more people become aware about HIV, the more they will ask questions - are scientists ready to answer the questions?' asks Petronella.

'You have to be honest with people', she continues. 'This is research and you don't always know what the outcome will be.'

\section{NATIONAL AND INTERNATIONAL PRESENCE}

Masikhulisane is facilitating three national processes - the development of a national $C A B$, the development of a national vaccine educator's forum, with a focus on capacity development and information sharing, and the formation of a SAAVI Community Working Group.

They are also working with the World Health Organization and UNAIDS to pull together the African AIDS Vaccine Programme Community Working Group and a meeting of community representatives from seven African countries.

'The relationship with WHO fits in so well with our own plans because we were planning on working much more closely with other African countries,' says Elise. 'We can learn from other countries but they can learn from us as well.'

A big challenge is to extend the programme's presence beyond the three provinces.

'Obviously we would like to work throughout the country', says Elise, 'but that would require major resources. I'm not that sure that that is necessarily one of the country's immediate priorities.'

The programme recently undertook a visit to all the trial sites and received support from the people working within the trial site communities and from the community representatives as well.

'Now we know we have support from the rest of the people doing this work,' says Elise.

'It's an exciting programme,' she continues. 'It's combining community development and community education experience with science and research, and that is challenging. To convince people that something like this can work could be a major challenge.' 